\title{
A UNIQUENESS THEOREM FOR FIXED POINTS
}

\author{
H. L. SMITH AND C. A. STUART
}

\begin{abstract}
In a recent paper, R. Kellogg [3] showed that if $F: \bar{D} \rightarrow \bar{D}$ is a completely continuous map of the closure of a bounded, convex, open set $D$ in a real Banach space $X, F \in C^{1}(D), 1$ is not an eigenvalue of $F^{\prime}(x)$ for $x \in D$, and $F(x) \neq x$ for $x \in \partial D$, then $F$ has a unique fixed point in $D$. More recently, $L$. Talman [7] extended this result to $k$-set contractions when $k<1$. The main result of this note is to show that, if the dimension of $X$ is larger than one, the result of Kellogg and its extension by Talman remain valid provided that the set $\{x \in D: 1$ is an eigenvalue of $F^{\prime}(x)$ \} has no accumulation points in $D$, the other assumptions remaining the same. This result is obtained as a corollary of a more general result which gives conditions under which the set of fixed points of $F$ in $D$ is connected.
\end{abstract}

In a recent paper, R. Kellogg [3] showed that if $F: \bar{D} \rightarrow \bar{D}$ is a completely continuous map of the closure of a bounded, convex, open set $D$ in a real Banach space $X, F \in C^{1}(D), 1$ is not an eigenvalue of $F^{\prime}(x)$ for $x \in D$, and $F(x) \neq x$ for $x \in \partial D$, then $F$ has a unique fixed point in $D$. The existence of a fixed point for $F$ follows from the well-known Schauder Fixed Point Theorem, thus the result in [3] establishes the uniqueness of the fixed point. More recently, L. Talman [7] extended this result to $k$-set contractions when $k<1$. Kellogg's theorem is also contained in a result of Berger [1, Theorem 5.4.7] where a simpler proof is given based on a more fundamental property of topological degree, namely the homotopy property. Both Kellogg and Talman base their proofs on the Leray-Schauder formula. The main result of this note is to show that, if the dimension of $X$ is larger than one, the result of Kellogg and its extension by Talman remain valid provided that the set $\left\{x \in D: 1\right.$ is an eigenvalue of $\left.F^{\prime}(x)\right\}$ has no accumulation points in $D$, the other assumptions remaining the same. The reader can easily construct counterexamples to this result when the dimension of $X$ is one. We obtain this result as a corollary of a more general result which gives conditions under which the set of fixed points of $F$ in $D$ is connected. A result of Krasnoselskii and Perov (see Theorem 13.4 of [8]) gives a similar conclusion under different hypotheses.

Before proceeding, we recall some basic definitions. If $B \subseteq X$ is a bounded set, the set measure of noncompactness of $B, \alpha(B)$, is defined by $\alpha(B)=\inf \{\varepsilon>0: B$ has a finite cover by sets whose diameters do not exceed $\varepsilon\}$. A function $F$ defined on a subset of $X$ is a $k$-set-contraction if $\alpha(F(B))<k \alpha(B)$ for all bounded subsets $B$ of the domain of $f$. Since a set $B$ is precompact if and only if $\alpha(B)=0$, it follows that every completely continuous map is a 0 -set-contraction. In what follows we

Received by the editors December 5, 1978 and, in revised form, June 5, 1979.

AMS (MOS) subject classifications (1970). Primary 47H10, 54H25, 55C20, 55C25.

Key words and phrases. Fixed point, $K$-set contraction. 
make use of the topological degree for $k$-set-contractive perturbations of the identity developed by R. Nussbaum [5].

Main results. Let $X$ be a real Banach space and let $D$ be a bounded open connected subset of $X$. Let $F: \bar{D} \rightarrow X$ be a continuous $k$-set-contraction for some $k<1$ which is continuously differentiable on $D$. Set $\psi=I-F$, where $I$ denotes the identity map on $X$, and let $S=\{x \in \bar{D}: \psi(x)=0\}$. Recall that $\psi$ is a proper map [2], that is, $\psi^{-1}(K)$ is compact in $\bar{D}$ whenever $K$ is compact in $X$, and that $\psi^{\prime}(x)$ is a Fredholm map of index zero for all $x \in D[6]$. Let

$$
R=\left\{x \in D: \psi^{\prime}(x): X \rightarrow X \text { is an isomorphism }\right\} .
$$

Since $\psi^{\prime}(x)$ is Fredholm of index zero,

$$
R=\left\{x \in D: 1 \notin P \sigma\left(F^{\prime}(x)\right)\right\}
$$

where $\operatorname{P\sigma }(A)$ denotes the point spectrum of $A$. By the inverse function theorem, if $x \in R$ there exist open subsets $U_{x}$ and $V_{x}$ of $X$ such that $x \in U_{x} \subset D, \psi(x) \in V_{x}$ and $\psi: U_{x} \rightarrow V_{x}$ is a homeomorphism. We can always assume that $V_{x}$ is a ball and that $\psi$ is injective on $\bar{U}_{x}$. Note that the set $R$ is open.

LEMMA. $\operatorname{deg}\left(\psi, U_{x}, \psi(x)\right)$ is constant on components of $R$.

Proof. It is enough to prove that $\operatorname{deg}\left(\psi, U_{x}, \psi(x)\right)$ is locally constant on $R$. Let $x_{0} \in R$ and let $y \in U_{x_{0}}$. Let $\omega=U_{x_{0}} \cap U_{y}$. Then $\omega$ is open and $\operatorname{deg}\left(\psi, U_{x_{0}}, \psi(y)\right)$ $=\operatorname{deg}(\psi, \omega, \psi(y))=\operatorname{deg}\left(\psi, U_{y}, \psi(y)\right)$ since the equation (for $\left.x\right) \psi(x)=\psi(y)$ has no solutions in $\bar{U}_{x_{0}} \backslash \omega$ and $\overline{U_{y} \backslash \omega}$ because $\psi$ is injective on $\bar{U}_{x_{0}}$ and $\bar{U}_{y}$. Therefore we need only prove that

$$
\operatorname{deg}\left(\psi, U_{x_{0}}, \psi\left(x_{0}\right)\right)=\operatorname{deg}\left(\psi, U_{x_{0}}, \psi(y)\right) .
$$

In order to accomplish this, set $H(x, t)=\psi(x)-t \psi(y)-(1-t) \psi\left(x_{0}\right)$ for $x \in$ $\bar{U}_{x_{0}}, t \in[0,1]$. Suppose $H(z, s)=0$ for some $z \in \partial U_{x_{0}}$ and $s \in[0,1]$. Then $\psi(z)=$ $s \psi(y)+(1-s) \psi\left(x_{0}\right) \in V_{x_{0}}$ since $V_{x_{0}}$ is a ball and $\psi(y), \psi\left(x_{0}\right) \in V_{x_{0}}$. But $\psi:$ $U_{x_{0}} \rightarrow V_{x_{0}}$ is a homeomorphism and so there exists $z_{0} \in V_{x_{0}}$ such that $\psi\left(z_{0}\right)=\psi(z)$. This contradicts our assumption that $\psi$ is injective on $\bar{U}_{x_{0}}$ and so $H$ is an admissible homotopy. Thus

$$
\begin{aligned}
\operatorname{deg}\left(\psi, U_{x_{0}}, \psi\left(x_{0}\right)\right) & =\operatorname{deg}\left(H(\cdot, 0), U_{x_{0}}, 0\right)=\operatorname{deg}\left(H(\cdot, 1), U_{x_{0}}, 0\right) \\
& =\operatorname{deg}\left(\psi, U_{x_{0}}, \psi(y)\right) .
\end{aligned}
$$

THEOREM. Suppose that

(1) $S \cap \partial D=\varnothing$ and $\operatorname{deg}(\psi, D, 0)= \pm 1$,

(2) $R$ is connected,

(3) $R$ is dense.

Then $S$ is nonempty and connected. If in addition, $S \cap R \neq \varnothing$ then $S$ is a singleton.

Proof. By (1), $S \neq \varnothing$ and, since $\psi$ is proper, $S$ is compact. If $S$ is not connected then there exist two nonempty compact sets $S_{1}$ and $S_{2}$ such that $S=S_{1} \cup S_{2}$ and $S_{1} \cap S_{2}=\varnothing$. Hence there exist open subsets $\Omega_{1}$ and $\Omega_{2}$ in $D$ such that $\bar{\Omega}_{1} \cap \bar{\Omega}_{2}=$ $\varnothing$ and $S_{i} \subset \Omega_{i}, i=1$ and 2 . Thus 


$$
\pm 1=\operatorname{deg}(\psi, D, 0)=\operatorname{deg}\left(\psi, \Omega_{1}, 0\right)+\operatorname{deg}\left(\psi, \Omega_{2}, 0\right)
$$

and so we may assume that $\operatorname{deg}\left(\psi, \Omega_{1}, 0\right) \neq 0$. Let $x_{2} \in S_{2}$. Then by (3) there exists a sequence $\left\{y_{k}\right\} \subseteq R$ such that $y_{k} \rightarrow x_{2}$ as $k \rightarrow \infty$. Thus there exist open sets $U_{k}$ and $V_{k}$ as above: $y_{k} \in U_{k}, \psi\left(y_{k}\right) \in V_{k}$ and $\psi: U_{k} \rightarrow V_{k}$ is a homeomorphism. We can, and do, assume that the diameter of $V_{k}$ tends to zero as $k \rightarrow \infty$. By Smale's generalization of Sard's theorem [4], each $V_{k}$ contains some points all of whose preimages in $D$ are in $R$. For each $k$, we select such a point $p_{k} \in V_{k}$. Now, $p_{k} \rightarrow 0$ as $k \rightarrow \infty$ and so by (1) we may assume that $\psi^{-1}\left(p_{k}\right) \cap \partial D=\varnothing$. Since $\psi$ is proper we can then conclude that $\psi^{-1}\left(p_{k}\right)$ contains a finite number, $n_{k}$, of elements and that

$$
\operatorname{deg}\left(\psi, D, p_{k}\right)=\sum_{x \in \psi^{-1}\left(p_{k}\right)} \operatorname{deg}\left(\psi, U_{x}, p_{k}\right)
$$

Now by the lemma, $\operatorname{deg}\left(\psi, U_{x}, \psi(x)\right)=l$ (a constant) on $R$ and $\operatorname{so} \operatorname{deg}\left(\psi, D, p_{k}\right)=$ $\ln _{k}$. Since $\operatorname{deg}\left(\psi, D, p_{k}\right)=\operatorname{deg}(\psi, D, 0)= \pm 1$ for all large $k$, this implies that $l= \pm 1$ and that $n_{k}=1$. Letting $\psi\left(z_{k}\right)=p_{k}$, then we have $z_{k} \in U_{k}$ and since the diameter of $V_{k} \rightarrow 0$ as $k \rightarrow \infty$ the same holds for $U_{k}$ so $z_{k} \rightarrow x_{2}$ as $k \rightarrow \infty$. It follows that $\psi^{-1}\left(p_{k}\right) \cap \bar{\Omega}_{1}=\varnothing$ for all large $k$ and hence $\operatorname{deg}\left(\psi, \Omega_{1}, p_{k}\right)=0$. Since

$$
\operatorname{deg}\left(\psi, \Omega_{1}, p_{k}\right)=\operatorname{deg}\left(\psi, \Omega_{1}, 0\right) \neq 0
$$

for large $k$ we have arrived at a contradiction. Thus $S$ must be connected.

If in addition $S \cap R \neq \varnothing$ then $S$ contains an isolated solution of $\psi(x)=0$, hence $S$ must be a singleton.

In general, $S$ is not a singleton. Let $X=R^{2}$ and $D=(-1,1) \times(-1,1)$. Let $F$ : $\bar{D} \rightarrow \bar{D}$ by $(x, y) \rightarrow\left(x\left(1-y^{2} / 2\right) g\left(x^{2}-1 / 4\right),-y\right)$ where $g: R \rightarrow R$ is a $C^{\infty}$ function satisfying $g(t)=1$ for $t \leqslant 0, g^{\prime}(t)<0$ for $t>0$ and $g(3 / 4)=1 / 2$. Then the hypotheses (1), (2) and (3) of the theorem are satisfied $\left(R^{c}=\{(x, 0): x \leqslant 1 / 2\}\right.$ and $\operatorname{deg}(I-F, D, 0)=+1)$ and $S=\{(x, 0): x<1 / 2\}$.

COROLlary 1. Suppose that

(a) $\bar{D}$ is convex

(b) $F(\bar{D}) \subset \bar{D}$ and $S \cap \partial D=\varnothing$,

(c) $D \backslash R$ has no accumulation points in $D$,

(d) $\operatorname{dim} X>1$.

Then $S$ is a singleton.

Proof. $S$ is compact and nonempty since, by (b) we can assume that $\operatorname{deg}(\psi, D, 0)$ $=+1$ (see [3]). If $\operatorname{dim} X>1$ then, by (c), $R$ is connected so the theorem applies. Hence $S$ is connected and either $S$ is a singleton or $S$ has an accumulation point which must lie in $S$. Since $D \backslash R$ has no accumulation points $S \cap R \neq \varnothing$. The result now follows from the theorem.

The following is a trivial example of a situation where the corollary applies but Kellogg's theorem fails. Let $D=\left\{(x, y) \in \mathbf{R}^{2}:\left(x^{2}+y^{2}\right)^{1 / 2}<2 / 3\right\}$ and $F(x, y)=$ $\left(x^{2}-y^{2}, 2 x y\right)=z^{2}$ where $z=x+i y$. Clearly $F: \bar{D} \rightarrow \bar{D}$ and $F(x, y) \neq(x, y)$ for $(x, y) \in \partial D$. The set $\left\{(x, y): 1\right.$ is an eigenvalue of $\left.F^{\prime}(x, y)\right\}=\{(1 / 2,0)\} \in D$ and $F$ has the unique fixed point $(0,0)$. 
Finally, we note that by approximation it is possible to remove the assumption that $F$ is differentiable in the theorem.

COROllary 2. Suppose that $\psi=I-F$ where $F$ is a continuous $k$-set-contraction for some $k<1$ on $\bar{D}$, with $D$ a bounded open subset of $X$. Suppose that

(a) $\operatorname{deg}(\psi, D, 0)= \pm 1$.

(b) There exists a sequence of continuously differentiable $k$-set-contractions $F_{n}$ on $\bar{D}$ such that $\psi_{n}=I-F_{n}$ converges to $\psi$ uniformly on $\bar{D}$.

(c) For all large $n, R_{n}=\left\{x \in D: \psi_{n}^{\prime}(x)\right.$ is an isomorphism $\}$ is connected and dense in $D$.

Then $S=\{x \in D: \psi(x)=0\}$ is connected.

Proof. Since the degree is continuous in its first and last arguments, there exist $r>0$ and a positive integer $N_{1}$ such that

$$
\operatorname{deg}\left(\psi_{n}-y, D, 0\right)=\operatorname{deg}(\psi, D, 0)= \pm 1
$$

for all $n \geqslant N_{1}$ and $|y|<r$. Hence, for each $n \geqslant N_{1}$ and $|y|<r, S_{n}(y)=\{x \in D$ : $\left.\psi_{n}(x)=y\right\}$ is connected.

We now proceed exactly as in the theorem letting $S=S_{1} \cup S_{2}, S_{1} \cap S_{2}=\varnothing$ and $S_{i} \subset \Omega_{i}, i=1,2$, where $\bar{\Omega}_{1} \cap \bar{\Omega}_{2}=\varnothing$. As before

$$
\pm 1=\operatorname{deg}\left(\psi, \Omega_{1}, 0\right)+\operatorname{deg}\left(\psi, \Omega_{2}, 0\right)
$$

so we may assume $\operatorname{deg}\left(\psi, \Omega_{1}, 0\right) \neq 0$. By choosing $r$ smaller and $N_{1}$ larger if necessary we have that $\operatorname{deg}\left(\psi_{n}, \Omega_{1}, y\right)$ is defined and not equal to zero for $n>N_{1}$ and $|y|<r$. Now fix $n_{1} \geqslant N_{1}$ so large that $\sup _{x \in \bar{D}}\left|\psi_{n_{1}}(x)-\psi(x)\right|<r$. Let $x_{2} \in S_{2}$ and $y=\psi_{n_{1}}\left(x_{2}\right)$. Then $|y|<r$, so that $S_{n_{1}}(y)$ is connected. But $S_{n_{1}}(y) \cap \Omega_{2} \neq \varnothing$ and $\operatorname{deg}\left(\psi_{n_{1}}, \Omega_{1}, y\right) \neq 0$ so $S_{n_{1}}(y) \cap \partial \Omega_{1} \neq \varnothing$ and $S_{n}(y) \cap \partial \Omega_{1}=\varnothing$. This contradicts that $S_{n_{1}}(y)$ is connected and proves the corollary.

In case $F$ has a fixed point $x_{0}$ which can be shown to be isolated (e.g. $F^{\prime}$ exists at $x_{0}$ and is invertible) then the set $S$ in Corollary 2 is a singleton.

\section{REFERENCES}

1. M. Berger, Nonlinearity and functional analysis, Academic Press, New York, 1977.

2. F. E. Browder, Nonlinear operators and nonlinear equations of evolution in Banach spaces, Proc. Sympos. Pure Math., Vol. 18, Amer. Math. Soc., Providence, R.I., 1976.

3. R. B. Kellogg, Uniqueness in the Schauder fixed point theorem, Proc. Amer. Math. Soc. 60 (1976), 207-210.

4. S. Smale, An infinite dimensional version of Sard's theorem, Amer. J. Math. 87 (1965), 861-866.

5. R. D. Nussbaum, The fixed point index and fixed point theorems for $k$-set-contractions, Ph.D. thesis, Univ. of Chicago, 1969.

6. ___ The radius of the essential spectrum, Duke Math. J. 37 (1970), 473-478.

7. L. A. Talman, A note on Kellogg's uniqueness theorem for fixed points, Proc. Amer. Math. Soc. 69. (1978), 248-250.

8. E. Zeidler, Vorlesungen über nichlinear Functionalanalysis. I, Fixpunktsatze, Teubner, Leipzig, 1976.

Department of Mathematics, Arizona State University, Tempe, Arizona 85281

département de Mathématiques, École Polytechnique Fédérale de lausanne, 61, avenue de Cour, CH-1007 Lausanne, Switzerland 